Review

\title{
New insights into meticillin-resistant Staphylococcus aureus (MRSA) pathogenesis, treatment and resistance ${ }^{i_{2}}$
}

\author{
Ian M. Gould ${ }^{\mathrm{a}, *}$, Michael Z. David ${ }^{\mathrm{b}}$, Silvano Esposito ${ }^{\mathrm{c}}$, Javier Garau ${ }^{\mathrm{d}}$, Gerard Lina ${ }^{\mathrm{e}}$, Teresita Mazzei ${ }^{\mathrm{f}}$, \\ Georg Peters ${ }^{g}$
}

a Department of Medical Microbiology, Aberdeen Royal Infirmary, Foresterhill, Aberdeen AB25 2ZN, UK

${ }^{\mathrm{b}}$ Departments of Medicine, Health Studies, and Pediatrics, University of Chicago, Chicago, IL, USA

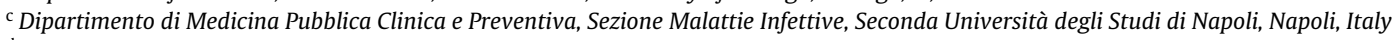

d Department of Medicine, Hospital Universitari Mutua de Terrassa, Terrassa, Barcelona, Spain

e Centre National de Référence des Staphylocoques, Université Lyon, INSERM, Hospices Civils de Lyon, Lyon, France

${ }^{\mathrm{f}}$ Department of Preclinical and Clinical Pharmacology, Florence, Italy

g Institute of Medical Microbiology, Münster, Germany

\section{A R T I C L E I N F O}

\section{Keywords:}

Drug resistance

Meticillin-resistant Staphylococcus aureus

Pneumonia

Staphylococcal skin infections

Virulence

\begin{abstract}
A B S T R A C T
Meticillin-resistant Staphylococcus aureus (MRSA) remains one of the principal multiply resistant bacterial pathogens causing serious healthcare-associated and community-onset infections. This paper reviews recent studies that have elucidated the virulence strategies employed by MRSA, key clinical trials of agents used to treat serious MRSA infections, and accumulating data regarding the implications of antibacterial resistance in MRSA for clinical success during therapy. Recent pre-clinical data support a species-specific role for Panton-Valentine leukocidin in the development of acute severe S. aureus infections and have elucidated other virulence mechanisms, including novel modes of internalisation, varying post-invasion strategies (featuring both upregulation and downregulation of virulence factors) and phenotypic switching. Recent double-blind, randomised, phase III/IV clinical trials have demonstrated the efficacy of linezolid and telavancin in hospital-acquired pneumonia (HAP) and complicated skin and skin-structure infections (cSSSIs) caused by MRSA. Tigecycline was non-inferior to imipenem/cilastatin in non-ventilator-associated HAP but was inferior in ventilator-associated pneumonia and has shown a higher rate of death than comparators on meta-analysis. Ceftaroline was clinically and microbiologically non-inferior to vancomycin/aztreonam in the treatment of MRSA cSSSI. Key resistance issues include a rise in vancomycin minimum inhibitory concentrations in MRSA, reports of clonal isolates with linezolid resistance mediated by acquisition of the chloramphenicol/florfenicol resistance gene, and case reports of daptomycin resistance resulting in clinical failure. Novel antimicrobial targets must be identified with some regularity or we will face the risk of untreatable S. aureus infections.
\end{abstract}

(C) 2011 Elsevier B.V. and the International Society of Chemotherapy. All rights reserved.

\section{Introduction}

Meticillin-resistant Staphylococcus aureus (MRSA) remains one of the principal multiply resistant bacterial pathogens causing complicated skin and skin-structure infections (cSSSI) and serious hospital-acquired infections, especially bloodstream infections (BSIs) and ventilator-associated pneumonia (VAP) [1-3]. Overall, MRSA is estimated to cause 171200 healthcare-associated

\footnotetext{
is This review is based on discussions held at the International Society of Chemotherapy (ISC) MRSA Consensus Meeting, 15-16 March 2011, Florence, Italy.

* Corresponding author. Tel.: +44 1224554 954; fax: +44 1224550632.

E-mail addresses: i.m.gould@abdn.ac.uk, Jacqueline.Cooper@nhs.net (I.M. Gould).
}

infections (HAIs) in Europe each year, corresponding to $44 \%$ of all HAIs. It is also estimated to cause 5400 attributable extra deaths and over a million extra days of hospitalisation associated with these infections [1]. Accumulating data indicate that MRSA infections are associated with a worse prognosis than meticillin-susceptible $S$. aureus (MSSA) infections [3-6]. Most recently, the BURDEN study, an international prospective cohort study, found that MRSA bacteraemia almost doubled the odds of 30-day mortality compared with MSSA bacteraemia [odds ratio (OR) $1.8 ; P=0.04$ ] and that both cohorts had elevated mortality and length of stay compared with controls [3]. The emergence of community-associated MRSA (CA-MRSA) is of considerable concern, although these remain less common in Europe than in the USA. Importantly, the simplistic distinction between CA-MRSA and healthcare-associated MRSA (HA-MRSA) infection has now been superseded, as CA-MRSA clones 
are now a potential cause of HA infections and community-onset infections in hospitalised patients as well as MRSA infections in the community, particularly in the USA [7-9].

Meticillin resistance in $S$. aureus results from acquisition of the mecA gene located within the mobile element known as the staphylococcal cassette chromosome mec (SCCmec). Until recently, eight SCCmec types were defined according to the SCCmec type and the chromosomal background determined by multilocus sequence typing [10]. HA-MRSA infections are generally caused by multidrug-resistant strains harbouring SCCmec types I, II and III, whereas CA-MRSA carry SCCmec types IV, V or VII. In Europe, sequence type 80 (ST80), SCCmec type IV is the predominant CAMRSA clone, whereas the ST8, SCCmec type IV clone exhibiting the USA300 pulsed-field gel electrophoresis profile predominates in the USA [8]. Whilst CA-MRSA are usually susceptible to most antibacterial classes, US300 strains with plasmid-mediated resistance to clindamycin, mupirocin, gentamicin, trimethoprim and/or doxycycline have been reported [11]. The novel SCCmec types IX and $\mathrm{X}$, type $\mathrm{V}(5 \mathrm{C} 2 \& 5)$ subtype $\mathrm{C}$ and type IVa have recently been described in clonal complex 398 MRSA strains [12].

This review is based on discussions held at an expert panel meeting sponsored by the International Society of Chemotherapy (ISC) in Florence, Italy, 15-16 March 2011. This paper adds to and updates previous statements by working groups from the ISC and the European Society of Clinical Microbiology and Infectious Diseases (ESCMID) [13,14]. It focuses on recent published data that have elucidated the virulence strategies employed by MRSA, on key clinical trials published since the last ESCMID/ISC statement, and on the accumulating data regarding the clinical implications of resistance during treatment for serious MRSA infections, principally in hospitalised patients.

\section{Pathogenesis: new insights into MRSA virulence}

Staphylococcus aureus is a versatile pathogen that can express an array of virulence factors, including adhesins [e.g. fibronectinbinding proteins (FnBPs) and protein A] that mediate binding to host cells, enzymes (e.g. proteases and lipases), toxins [e.g. $\alpha$-haemolysin and Panton-Valentine leukocidin (PVL)], phenolsoluble modulins and capsular polysaccharides. Expression of these virulence factors is controlled by complex staphylococcal regulatory networks, including the accessory gene regulator (agr) system, and these genes vary between strains. Recent data have shed light on the role of PVL, an important yet controversial virulence factor. PVL is a bicomponent pore-forming exotoxin produced by $2-3 \%$ of clinical S. aureus isolates [15] and is almost universally present in CA-MRSA clones [8]. Clinical evidence suggests that PVL is associated with acute, severely necrotising skin infections and pneumonia $[16,17]$ and with a greater systemic inflammatory response and more severe local disease in osteomyelitis [18]. However, the pathogenic role of PVL has been questioned following a lack of observable effects in mouse models of infection.

Evidence that the effects of PVL are species-specific may explain the seemingly contradictory evidence regarding its importance in the pathogenesis of MRSA infections: PVL induces rapid activation and cell death in human and rabbit neutrophils, but not in murine or simian cells $[19,20]$. Studies in rabbit models have confirmed that PVL expression in the S. aureus USA300 strain is associated with more severe skin infection lesions compared with non-PVL strains [20]. In a rabbit osteomyelitis model, PVL expression was associated with greater persistence of infection and with a significantly greater likelihood of bone deformation and extension of the infection to muscle and joints [21]. Evidence from a pneumonia model suggests that PVL causes lung necrosis by activating and lysing polymorphonuclear leukocytes [22].
New insights have also been gained into the pathogenic mechanisms involved in S. aureus endovascular infections (e.g. endocarditis, thrombophlebitis, and catheter-related or vascular graft infections). Pathogenic strains attach to host endothelial cells via adhesins before invading and triggering inflammatory responses and cell death. Recent studies have also revealed a novel FnBP-independent mechanism of staphylococcal internalisation mediated in S. aureus and Staphylococcus epidermidis by the autolysin/adhesins Atl and AtlE, respectively, and by heat shock protein as the host cell surface receptor [23]. It is now clear that strains differ in their post-invasion strategies and that this explains the different disease processes observed. Highly virulent strains such as 6850 and ST239 express toxins and other virulence factors following cell invasion, regulated by the agr system. In contrast, others (e.g. Cowan I) persist within intact cells without causing dramatic inflammatory reactions or acute cytotoxicity owing to a defective agr system [24]. These examples of downregulation of certain virulence factors may represent alternate strategies whereby such strains can result in persistent or recurrent chronic infections.

Furthermore, S. aureus appears to switch between phenotypes according to its location. Small colony variant (SCV) phenotypes of $S$. aureus are associated with persistent infections such as osteomyelitis and endocarditis. Adapted for intracellular persistence, SCV strains are characterised by slow growth and reduced expression of most extracellular virulence factors [25]. Highly virulent wild-type isolates can also persist intracellularly and develop SCV-like phenotypes, thereby representing a reservoir for chronic refractory infections. These phenotypes then revert to the fully virulent wild-type form when leaving the intracellular location and infecting new cells [26].

\section{Clinical trials}

Choice of antimicrobial therapy for suspected severe MRSA infections should be based primarily on the local prevalence and resistance profile of MRSA and on the risk factors and clinical characteristics present in individual patients. In a previous statement by the ESCMID/ISC MRSA Working Group, vancomycin was recommended as the standard option for the initial treatment of MRSA bacteraemia and endocarditis, in particular when sepsis is not life-threatening and the infecting strain has a minimum inhibitory concentration (MIC) of $\leq 1 \mathrm{mg} / \mathrm{L}$. A semisynthetic penicillin should be added to cover MSSA in severe sepsis. A change to daptomycin was recommended in cases where there is a slow response to vancomycin or in complicated bacteraemia, relapse or breakthrough MRSA bacteraemia by organisms with a vancomycin MIC $>1.0 \mathrm{mg} / \mathrm{L}$. Daptomycin may be preferred for first-line initial therapy in patients with life-threatening sepsis or renal impairment and when the vancomycin MIC is $>1.0 \mathrm{mg} / \mathrm{L}$ and in patients who have previously received optimally conducted glycopeptide therapy. Linezolid was recommended for first-line empirical therapy for suspected MRSA VAP and hospital-acquired pneumonia (HAP), especially in patients with recent vancomycin exposure, when the vancomycin MIC is $>1 \mathrm{mg} / \mathrm{L}$ or is considered likely to be elevated, and in patients with renal failure [14]. Recent guidelines issued by the Infectious Disease Society of America (IDSA) recommend vancomycin or daptomycin for bacteraemia and endocarditis (plus rifampicin for prosthetic valve infections) and vancomycin, linezolid or clindamycin for HA-MRSA or CA-MRSA pneumonia [27], with the choice depending on local resistance profiles of clinical MRSA isolates. Options for empirical treatment of cSSSIs in hospitalised patients include vancomycin, linezolid, daptomycin, telavancin (not yet available in Europe) or clindamycin $[27,28]$. Options for empirical treatment of CA-MRSA SSSIs in outpatients 
include oral clindamycin, trimethoprim/sulfamethoxazole (SXT), a tetracycline or linezolid [27].

The following section reviews recent clinical studies and metaanalyses of recommended and investigational agents for MRSA infections.

\subsection{Linezolid}

On meta-analysis, linezolid has shown efficacy similar to comparators in the treatment of Gram-positive pneumonia and bacteraemia $[29,30]$. Two recent meta-analyses did not show a significant benefit of linezolid over glycopeptides in terms of clinical success, microbiological eradication or mortality in the treatment of HAP, including MRSA infections [31,32].

Results from the randomised, double-blind, multicentre, phase IV, ZEPHyR study of linezolid [600 mg intravenous (i.v.) every $12 \mathrm{~h}$ (q12 h)] versus vancomycin [15 mg/kg i.v. q12 h with therapeutic drug monitoring (TDM)] in MRSA culture-proven HAP have recently been reported in abstract form [33]. This was a non-inferiority trial with a nested superiority hypothesis. Clinical success rates at the end of the study (7-30 days after the end of therapy; primary endpoint) in the per-protocol (PP) group were $57.6 \%$ for linezolid and $46.6 \%$ for vancomycin (Table 1). Linezolid was non-inferior and statistically superior $(P=0.042)$ to vancomycin according to this endpoint and according to rates of clinical and microbiological success at the end of therapy, respectively. The 60-day mortality rate was similar between the groups (94 deaths in the linezolid group and 100 in the vancomycin group), but the study was not powered to compare these rates. These preliminary data show a significant benefit of linezolid over vancomycin in HAP due to MRSA, although the clinical success rates remain low. Subanalyses of the results in patients with VAP and those with bacteraemia would be useful.

Several recent meta-analyses have shown significantly superior clinical and/or microbiological success rates for linezolid versus vancomycin for MRSA-confirmed cSSSIs [30,34-36]. A large, international, open-label, phase IV trial compared linezolid with vancomycin $(15 \mathrm{mg} / \mathrm{kg}$ i.v. q12 h, adjusted to trough levels) in patients with MRSA-confirmed cSSSI $(n=1052)$ [37]. This was a non-inferiority study with a nested superiority test. In terms of clinical success, linezolid was non-inferior to vancomycin in the PP population (primary analysis) at the end-of-study assessment, with rates of $191 / 227(84 \%)$ versus $167 / 209(80 \%)(P=0.249)$ at the end of treatment. Linezolid was significantly superior to vancomycin in the modified intention-to-treat (mITT) population at the end of the study assessment [223/276 (81\%) vs. 196.266 (74\%); $P=0.048]$. Microbiologically, linezolid was significantly better than vancomycin in the PP population at the end of treatment and had similar efficacy at the end-of-study assessment. Linezolid was also associated with a shorter duration of hospitalisation and i.v. therapy [37].

According to meta-analyses, overall rates of adverse events (AEs) and discontinuations are similar for linezolid and vancomycin $[30,32,35]$. Nephrotoxicity is more common with vancomycin according to some meta-analyses [30,35], although not all [31]. Gastrointestinal effects and thrombocytopenia are more common with linezolid $[31,35]$.

\subsection{Daptomycin}

Daptomycin (Cubicin ${ }^{\circledR}$; Novartis) is a lipopeptide agent approved in Europe for the treatment of cSSSIs, right-side endocarditis and $S$. aureus bacteraemia in adults [38]. It is not effective in (or approved for) pneumonia therapy owing to its inactivation by lung surfactants.

According to a pre-specified subset analysis of a randomised trial, daptomycin $(6 \mathrm{mg} / \mathrm{kg} /$ day $)$ was as effective as vancomycin plus gentamicin in patients (total $n=88$ ) with MRSA bacteraemia or endocarditis [39]. More recently, data on 38 cancer patients with catheter-related BSI caused by Gram-positive bacteria treated with daptomycin were matched with data for historical controls treated with vancomycin [40]; $68 \%$ of patients in the daptomycin group and $80 \%$ in the vancomycin group had organisms with vancomycin MICs of 1-2 mg/L. Results from this small study suggest that daptomycin may be associated with improved 48 -h clinical and microbiological response, improved overall response and lower risk of nephrotoxicity, although the two groups were comparable with respect to length of hospital stay and death.

The approved dose of daptomycin is $4 \mathrm{mg} / \mathrm{kg}$ once daily in nonbacteraemic cSSSI and $6 \mathrm{mg} / \mathrm{kg}$ once daily in bacteraemic cSSSI and endocarditis [38]. Use of a $6 \mathrm{mg} / \mathrm{kg} /$ day dose has also been recommended in other circumstances, including rapidly progressing SSSI, sepsis syndrome, osteomyelitis and infections caused by vancomycin-intermediate S. aureus (VISA) [41]. Some experts recommend the use of even higher dosages of daptomycin, i.e. $8-10 \mathrm{mg} / \mathrm{kg}$ once daily, in order to reduce the risk of resistance selection during therapy (discussed below) [27]. The effectiveness and tolerability of daptomycin at these doses is supported by retrospective registry data $[42,43]$ and case series studies $[44,45]$. In one case series, 22 patients received daptomycin at a standard dose (mean $5 \mathrm{mg} / \mathrm{kg} /$ day) and 31 received a higher dose (mean $8 \mathrm{mg} / \mathrm{kg} /$ day, range $7-9 \mathrm{mg} / \mathrm{kg}$ ), mostly for the treatment of $S$. aureus bacteraemia or cSSSI [44]. The median treatment duration was 13.5 days and 19 days for the standard and high-dose groups, respectively. The higher dose was associated with higher rates of clinical success ( $94 \%$ vs. $73 \%$, respectively; $P=0.05$ ) and microbiological success (93\% vs. $68 \%$, respectively; $P<0.05$ ), with no clear increase in toxicity [44]. Recent data from 270 patients within a European prospective registry indicate that the rates of AEs and serious AEs were similar in patients treated with daptomycin doses of $\geq 8 \mathrm{mg} / \mathrm{kg}(n=270)$ and $<8 \mathrm{mg} / \mathrm{kg}(n=3330)$.

\subsection{Tigecycline}

Tigecycline (Tygacil ${ }^{\circledR}$; Wyeth), a bacteriostatic glycylcycline, is approved in the USA for the treatment of adults with cSSSI, complicated intra-abdominal infections (IAIs) and community-acquired pneumonia (CAP) [46] and in Europe for cSSSIs and IAIs [47].

Results were recently published from a large, phase III, multicentre, randomised, double-blind study that compared tigecycline (100 mg i.v., then $50 \mathrm{mg} \mathrm{q12} \mathrm{h}$ ), plus optional adjunctive therapy with ceftazidime [ $2 \mathrm{~g}$ i.v. every $8 \mathrm{~h}$ (q8h)] for Pseudomonas aeruginosa coverage, versus the carbapenem imipenem/cilastatin (0.5-1 g i.v. q8 h) plus optional adjunctive therapy with vancomycin ( $1 \mathrm{~g}$ i.v. q12 h) for MRSA coverage in patients with HAP $(n=945)$ [48]. The co-primary efficacy endpoints were clinical response at the test-of-cure assessment for the clinically evaluable and clinical mITT populations. Overall, tigecycline was non-inferior to imipenem/cilastatin in the mITT population $(62.7 \%$ and $67.6 \%$, respectively)(Table 1 ). However, tigecycline was inferior to the carbapenem in the clinically evaluable population (67.9\% vs. $78.2 \%$, respectively) (Table 1 ) owing to lower efficacy in the subset of patients with VAP. Indeed, tigecycline did not meet the criteria for non-inferiority in VAP patients in either the clinically evaluable or mITT populations. Tigecycline gave a clinical cure in $8 / 17$ patients (47.1\%) with non-VAP MRSA infection and $4 / 10$ patients $(40.0 \%)$ with MRSA VAP; the corresponding rates in the imipenem/cilastatin arm were $14 / 19$ (73.7\%) and 9/11 (81.8\%), respectively. The overall mortality rate was similar in the two arms, but the mortality rate in VAP patients was higher in the tigecycline arm. Exploratory analyses could not explain the difference in efficacy in VAP/non-VAP patients, although mechanical ventilation alone did not appear to be causative per se. The authors speculated 
Table 1

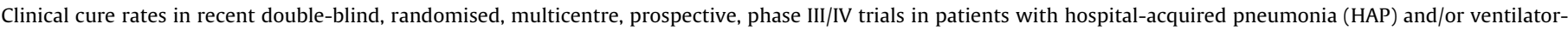
acquired pneumonia (VAP).

\begin{tabular}{|c|c|c|c|c|}
\hline Study/population & Infection/test & Treatment (dose) & & Difference $(95 \% \mathrm{CI})$ \\
\hline ZEPHyR [33] & MRSA-positive NP & $\begin{array}{l}\text { Linezolid ( } 600 \mathrm{mg} \text { i.v. } \\
\text { q12 h) }\end{array}$ & Vancomycin (15 mg/kg i.v. q12 h) & \\
\hline $\mathrm{PP}$ & EOS & $57.6 \%(95 / 165)$ & $46.6 \%(81 / 174)$ & $(0.5-21.6) ; P=0.042$ \\
\hline ATTAIN $1 \& 2$ (pooled) [54] & Gram-positive HAP & $\begin{array}{l}\text { Telavancin }(10 \mathrm{mg} / \mathrm{kg} \\
\text { i.v. q24 h) }\end{array}$ & Vancomycin (1 g i.v. q12 h) & \\
\hline All treated & TOC & $58.9 \%(441 / 749)$ & $59.5 \%(449 / 754)$ & $-0.7(-5.6$ to 4.3$)$ \\
\hline $\mathrm{CE}$ & TOC & $82.4 \%(257 / 312)$ & $80.7 \%(276 / 342)$ & $1.7(-4.3$ to 7.7$)$ \\
\hline ME: MRSA & TOC & $74.8 \%(104 / 139)$ & $74.7 \%(115 / 154)$ & $0.4(-9.5$ to 10.4$)$ \\
\hline Study $311[48]$ & HAP (including VAP) & $\begin{array}{l}\text { Tigecycline }(100 \mathrm{mg} \\
\text { i.v., then } 50 \mathrm{mg} \mathrm{q} 12 \mathrm{~h})^{\mathrm{a}}\end{array}$ & Imipenem/cilastatin (0.5-1 g i.v. q8 h $)^{\mathrm{a}}$ & \\
\hline \multirow[t]{3}{*}{ c-mITT } & TOC: all patients & $62.7 \%(276 / 440)$ & $67.6 \%(290 / 429)$ & $\begin{array}{l}-4.8(-11.0 \text { to } 1.3) ; \text { non-inferiority } \\
\text { test, } P=0.001\end{array}$ \\
\hline & TOC: VAP & $46.5 \%(37.6-55.5 \%)$ & $57.8 \%(48.2-66.9 \%)$ & $-11.3(-24.6$ to 2.0$)$ \\
\hline & TOC: non-VAP & $69.3 \%(63.9-74.4 \%)$ & $71.2 \%(65.9-76.2 \%)$ & $-1.9(-9.4$ to 5.6$)$ \\
\hline \multirow[t]{3}{*}{ CE } & TOC: all patients & $67.9 \%(182 / 268)$ & $78.2 \%(190 / 243)$ & $\begin{array}{l}-10.4(-17.8 \text { to }-3.0) \text {; non-inferiority } \\
\text { test, } P=0.120\end{array}$ \\
\hline & TOC: VAP & $47.9 \%(36.1-60.0 \%)$ & $70.1 \%(57.7-80.7 \%)$ & $-22.2(-37.8$ to -4.9$)$ \\
\hline & TOC: non-VAP & $75.4 \%(68.7-81.3 \%)$ & $81.3 \%(74.7-86.7 \%)$ & $-5.9(-14.5$ to 3.0$)$ \\
\hline
\end{tabular}

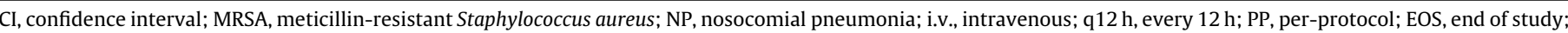
q24 h, every $24 \mathrm{~h}$; TOC, test of cure; CE, clinically evaluable; ME, microbiologically evaluable; q8 h, every $8 \mathrm{~h}$; c-mITT, clinical modified intention-to-treat.

a Patients treated with tigecycline could receive optional adjunctive therapy with ceftazidime ( $2 \mathrm{~g}$ i.v. q8 h) for Pseudomonas aeruginosa. Patients treated with imipenem/cilastatin could receive adjunctive therapy with vancomycin ( $1 \mathrm{~g}$ i.v. q12 h) for MRSA coverage.

that the results may be explained by an insufficient area under the concentration-time curve (AUC) exposure to tigecycline with the dose used [48].

Another group recently published a meta-analysis of eight randomised, phase III trials of tigecycline $(n=4651)$ in the management of cSSSI, CAP and IAI, seven of which were double-blind [49]. There were no statistically significant differences between tigecycline and comparators in clinical success rates in clinically evaluable patients overall or in cSSSI or CAP. Tigecycline was significantly less efficacious than comparators, however, in the treatment of IAI in evaluable patients [OR $=0.80,95 \%$ confidence interval $(\mathrm{CI})$ $0.65-0.98 ; P=0.03$ ] and overall there was a trend for lower efficacy for tigecycline in the clinical mITT population $(\mathrm{OR}=0.86,95 \%$ CI $0.74-1.01 ; P=0.06$ ).

Tigecycline gave numerically higher microbiological eradication rates than comparators against MRSA, but numerically lower eradication rates against MSSA, although no differences were significant. Tigecycline was associated with significantly more AEs (especially gastrointestinal events) than comparators as well as a non-significantly higher mortality rate [49]. Meta-analyses by the European Medicines Agency (EMEA) [50] and the manufacturer of tigecycline [51] have also documented a numerically higher incidence of death amongst tigecycline recipients in phase III and IV trials compared with comparator arms. The cause of this increased mortality risk has not been established.

Patients who develop superinfections, in particular HAP, appear to be associated with poorer outcomes and should be monitored accordingly. In February 2011, the EMEA Committee for Medicinal Products for Human Use (CHMP) recommended that the tigecycline label should be amended to warn of the mortality risk and to specify that tigecycline should only be used in situations where it is known or suspected that other alternatives are not suitable [50]. The US label for tigecycline was amended in 2010 to warn of this issue [46].

\subsection{Telavancin}

Telavancin (Vibativ ${ }^{\circledR}$; Astellas), a bactericidal lipoglycopeptide, is approved in the USA for the treatment of cSSSI [52]. In phase III trials, telavancin was clinically and bacteriologically non-inferior to vancomycin in patients $(n=579)$ with MRSA cSSSI [53]. Cure rates against MRSA infection were $91 \%$ for telavancin and $86 \%$ for vancomycin (95\% CI for the difference, $-1.1 \%$ to $9.3 \%$ ). Microbiological eradication rates were $90 \%$ and $85 \%$, respectively $(95 \% \mathrm{CI}$ for the difference, $-0.9 \%$ to $9.8 \%$ ). More recently, a meta-analysis showed significantly superior success rates for telavancin versus vancomycin for MRSA-confirmed cSSSIs [36].

Results have recently been reported from the two double-blind, randomised, phase III, non-inferiority ATTAIN 0015 and 0019 studies, which compared telavancin $(10 \mathrm{mg} / \mathrm{kg}$ i.v. every $24 \mathrm{~h})$ with vancomycin ( $1 \mathrm{~g}$ i.v. q12 h) in a total of 1503 patients with Grampositive HAP [54]. Vancomycin levels were measured at some sites, according to local protocols; the lack of these data from all sites complicates the interpretation of the study results. The primary efficacy analysis was a non-inferiority analysis of clinical response at follow-up/test of cure in each study. In addition, there was a pre-specified secondary superiority analysis in pooled data from patients with MRSA pneumonia in the two studies. Overall, ca. $50 \%$ of the population were aged $\geq 65$ years and $58 \%$ in both treatment arms were in intensive care at baseline. Approximately one-quarter had an Acute Physiology and Chronic Health Evaluation (APACHE) score of $\geq 20$ and $6 \%$ had bacteraemia. Telavancin was non-inferior to vancomycin in each of the studies and in the pooled all-treated population, with pooled cure rates of $58.9 \%$ vs. $59.5 \%$, respectively (Table 1 ). Telavancin and vancomycin gave similar cure rates in MRSA infections overall (74.8\% vs. 74.7\%). According to post hoc analysis, telavancin gave a statistically higher cure rate than vancomycin against all monomicrobial $S$. aureus infections ( $84.2 \%$ vs. $74.3 \%$, respectively; $95 \% \mathrm{CI}$ for the difference, $0.7-19.1 \%$ ) and a numerically higher cure rate against monomicrobial MRSA infections ( $81.8 \%$ vs. $74.1 \%$, respectively) and monomicrobial MSSA infections ( $87.9 \%$ vs. $75.0 \%$, respectively).

Telavancin was significantly more effective than vancomycin in monomicrobial $S$. aureus infections with a vancomycin MIC $\geq 1 \mu \mathrm{g} / \mathrm{mL}$ (87.1\% vs. $74.3 \% ; P=0.03$ ). Vancomycin gave a nonsignificantly higher cure rate overall in patients with mixed Grampositive/Gram-negative infection (66.2\% vs. 79.4\%). Cure rates were similar between the groups amongst patients with mixed infections who received adequate Gram-negative coverage. However, 'adequate' was not defined. Moreover, piperacillin/tazobactam or aztreonam were used for Gram-negative coverage and the former also covers MSSA. Overall rates of death and AEs were similar 
between the groups. The most common treatment-emergent AEs in both arms were diarrhoea, anaemia, hypokalaemia, constipation and renal impairment. Potentially clinically significant increases in serum creatinine levels were numerically more common in the telavancin group than in the vancomycin group (16\% vs. $10 \%$, respectively), as were serious AEs ( $31 \%$ vs. $26 \%$, respectively) and AEs leading to discontinuation of study medication ( $8 \%$ vs. $5 \%$, respectively). The most common serious AEs in both groups were septic shock, respiratory failure and multiorgan failure [54].

Evidence of a risk of acute renal failure caused by telavancin has prompted the CHMP to recommend limited approval of telavancin in Europe for the treatment of adults with HAP, including VAP, known or suspected to be caused by MRSA only in situations where it is known or suspected that other alternatives are not suitable [55]. An application for the indication of cSSSI in Europe has been withdrawn.

\subsection{Ceftaroline}

Ceftaroline, the active component of the pro-drug ceftaroline fosamil (Teflaro ${ }^{\circledR}$; Forest), is an advanced-generation cephalosporin that is approved in the USA for acute bacterial SSSI and CAP [56] but which is not yet approved in Europe.

Results were recently published from two identical, international, randomised, double-blind, phase III trials known as CeftAroliNe versus VAncomycin in Skin and Skin Structure Infections (CANVAS) 1 [57] and CANVAS 2 [58], together with a pooled analysis of these studies [59]. Adults with cSSSI were randomised to treatment with ceftaroline (600 mg i.v. q12 h; $n=693$ ) or vancomycin ( $1 \mathrm{~g} \mathrm{q} 12 \mathrm{~h}$, adjusted according to local policies) plus aztreonam ( $1 \mathrm{~g} \mathrm{q} 12 \mathrm{~h}$ ). Ceftaroline was clinically and microbiologically non-inferior to vancomycin/aztreonam in PP and ITT analyses, in the individual trials and the pooled analysis, including in mixed and polymicrobial infections. On pooled analysis, the two regimens gave similar clinical cure rates against MRSA in evaluable patients [142/152 (93.4\%) for ceftaroline and 115/122 (94.3\%) for vancomycin/aztreonam] as well as in the ITT population and against infections caused by MSSA. A trend for a lower rate of clinical cure in patients with bacteraemia is of unclear significance owing to the small number of cases. Amongst patients with MRSA bacteraemia, cure rates were $6 / 7$ (85.7\%) for ceftaroline and $2 / 2$ (100.0\%) for vancomycin/aztreonam [58]. Rates of AEs, serious AEs and discontinuations were similar between the groups. Vancomycin plasma level data were not reported, complicating evaluation of the results.

\subsection{Investigational agents}

A regulatory submission for the developmental lipoglycopeptide oritavancin for cSSSIs was declined by the US Food and Drug Administration (FDA) owing to insufficient data, and an EMEA application was subsequently withdrawn [60]. Results of the multicentre, double-blind, randomised, phase II SIMPLIFI study (reported in abstract form) suggest that oritavancin is similarly efficacious against Gram-positive cSSSI (including MRSA) when administered as a single dose $(1200 \mathrm{mg}$ ) or infrequently (i.e. $800 \mathrm{mg}$ on Day 1 , with an optional $400 \mathrm{mg}$ dose on Day 5) compared with daily dosing (200 $\mathrm{mg}$ for 3-7 days) [61].

Other agents in clinical development include nemonoxacin (TG873870), a novel non-fluorinated quinolone with in vitro and in vivo activity covering MRSA [62,63], and omadacycline (PTK796), an aminomethycycline structurally related to the tetracyclines [64]. Something should be said about dalbavancin. An (American) Advisory Board on the development of dalbavancin was held in Milan after the 21st European Congress of Clinical Microbiology and Infectious Diseases (ECCMID) (2011), indicating that clinical trials are in progress or contemplated very seriously. We could try to see whether something has been reported on this drug.

\subsection{Older agents}

Trimethoprim is commonly used in the management of uncomplicated skin abscesses caused by CA-MRSA, although the benefit of antibiotic therapy in this setting is not well characterised. Recently, a multicentre, double-blind, randomised, placebo-controlled trial has evaluated whether use of antibiotic therapy with oral SXT for uncomplicated SSSI reduces the rate of treatment failures during the 7 days after incision and drainage and the rate of new lesion formation within 30 days [65]. There was a similar incidence of treatment failure in patients receiving SXT $(15 / 88 ; 17 \%)$ and placebo $(27 / 102 ; 26 \%)(P=0.12)$. However, the 30 -day rate of new lesions was significantly lower in patients treated with the antibiotic (4/46; $9 \%$ ) versus placebo (14/50; $28 \%$ ) (difference, $19 \%$, $95 \% \mathrm{CI}$ of the difference, $4-34 \% ; P=0.02$ ). Therefore, antibiotic therapy may not improve treatment success rates in patients but may reduce the risk of subsequent lesions. Data are awaited from an ongoing randomised, open-label study comparing SXT and vancomycin in invasive MRSA infections in Israel (Clinicaltrials.gov identifier NCT00427076).

Other studies underway include a European trial comparing SXT plus rifampicin against linezolid for MRSA infections (NCT00711854) and a large US study of SXT, clindamycin or placebo within the outpatient management of uncomplicated SSTIs, including CA-MRSA, in children and adults (NCT00730028).

In contrast to previous findings, data from a small, randomised study in South Korea suggest that rifampicin ( $300 \mathrm{mg}$ orally twice daily) might provide benefit when added to vancomycin ( $1 \mathrm{~g}$ i.v. twice daily) in the management of confirmed MRSA HAP [66]. The clinical cure rate in the mITT population was significantly higher in patients treated with vancomycin/rifampicin (53.7\%; 22/41) compared with vancomycin monotherapy $(13 / 42 ; 31.0 \%)(P=0.047)$ as was the rate of 60 -day mortality $(50.0 \%$ vs. $26.8 \% ; P=0.042)$. The very small numbers of patients should be emphasised.

\section{Resistance, clinical failure and dosing}

\subsection{Vancomycin}

Vancomycin has long been the gold-standard agent for the empirical management of serious MRSA infections in hospitalised patients $[13,14,27,28]$. However, it has well-recognised limitations, including slow cidality, poor activity against MSSA, uncertainty regarding the prevalence of heterogeneous VISA (hVISA) strains, variations between and within patients in tissue distribution, and the need for TDM to ensure adequate levels and to minimise the risk of nephrotoxicity [67]. Although vancomycin remains active against MRSA [68,69], rising MICs within the susceptible range (MIC creep or leap) is a concern [14]. International data from the Tigecycline Evaluation and Surveillance Trial (T.E.S.T.) involving 20004 $S$. aureus isolates show that the proportion of MRSA with vancomycin MICs $\geq 2 \mathrm{mg} / \mathrm{L}$ increased from $5.6 \%$ in 2004 to $11.1 \%$ in $2009(P<0.001)$. The proportion of MSSA isolates with vancomycin MICs $\geq 2 \mathrm{mg} / \mathrm{L}$ rose from $2.6 \%$ in 2004 to $5.6 \%$ in $2009(P<0.001)$ [70]. Strains with high-level vancomycin resistance (acquired via the van A plasmid) remain very rare, although cases have been reported in the USA [71,72], India [73] and Iran [74]. The likelihood of reduced vancomycin susceptibility is significantly increased by recent prior vancomycin use [75].

MRSA strains with a vancomycin MIC of $2 \mathrm{mg} / \mathrm{L}$ have been associated in some prospective multivariate analyses with an increased risk of treatment failure and even an increased mortality rate in 
bacteraemic patients compared with strains with a lower MIC. Soriano et al. [76] prospectively evaluated the effect of vancomycin MIC on outcome of vancomycin therapy in 414 patients with MRSA bacteraemia. Multivariate analysis selected receipt of empirical vancomycin and an isolate with a vancomycin MIC of $2 \mathrm{mg} / \mathrm{L}$ $(\mathrm{OR}=6.39,95 \% \mathrm{CI} 1.68-24.3)$ as significant independent predictors of mortality [76]. Recent retrospective analyses suggest that VISA strains may contribute to vancomycin treatment failure and death in patients with MRSA bacteraemia, whereas heteroresistance may only reduce response rates without affecting mortality [77-79].

With regard to pharmacokinetics, vancomycin should be dosed to achieve a trough plasma concentration of $15-20 \mathrm{mg} / \mathrm{L}[14,27,67]$. In Scotland, Thomson et al. [80] used a population pharmacokinetic model to derive new dosage guidelines predicted to achieve a target trough of $10-15 \mathrm{mg} / \mathrm{L}$ earlier (using loading doses) and more consistently than previous dosage guidelines and to attain a satisfactory $\mathrm{AUC}_{24} / \mathrm{MIC}$ ratio in $87 \%$ of patients. TDM measurements report the total vancomycin level rather than levels of free (i.e. nonprotein bound) active drug. Recent data have confirmed that free vancomycin levels are highly variable both within and between patients and cannot be predicted from total vancomycin levels [81] This raises important questions about how vancomycin should be used and how TDM can be optimised. There are insufficient data to support a recommendation on continuous vancomycin infusion [14], although this mode of administration is considered unlikely to improve clinical outcomes [67].

Recommendations for the management of MRSA infections refractory to vancomycin treatment, in addition to necessary drainage or surgical debridement, include the use of high-dose daptomycin ( $10 \mathrm{mg} / \mathrm{kg} /$ day) if the isolate is susceptible, possibly in combination with another agent (e.g. gentamicin, rifampicin, linezolid, SXT or a $\beta$-lactam) [27].

\subsection{Linezolid}

Linezolid resistance was initially reported to occur most commonly as a result of a G2576T mutation in the drug target site, primarily the rRNA of the large ribosomal subunit [82]. This resistance has appeared only sporadically and is usually mediated by the presence of mutations in one or more alleles of the target 23S rRNA gene [83]. In 2008, linezolid-resistant isolates were isolated from 12 patients in a Spanish hospital [84,85]. These isolates remained susceptible to SXT, glycopeptides, tigecycline and daptomycin. Instead of the previously reported G2576T mutation, these clonal isolates were resistant owing to their possession of the chloramphenicol/florfenicol resistance ( $c f r$ ) gene. The $c f r$ gene mediates methylation of the 23S rRNA subunit and affects the binding of chloramphenicol, lincosamides (e.g. clindamycin), pleuromutilins and streptogramin A. This gene is thought to have been transmitted horizontally from coagulase-negative staphylococci [85]. Staphylococcus aureus and $S$. epidermidis isolates with cfr-mediated linezolid resistance have also been identified in the USA [68]. This mechanism is of concern owing to the potential for transmissibility and because it could threaten the effectiveness of other antibacterials that act against protein synthesis.

\subsection{Daptomycin}

Daptomycin resistance in wild-type MRSA is rare but is certainly of clinical concern, hence microbiology laboratories must provide daptomycin MICs in certain situations if daptomycin is to be used. Stepwise incubation of MRSA in increasing concentrations of daptomycin can increase the MICs of both daptomycin and vancomycin [86,87]. Although daptomycin is active against most hVISA and many VISA [88], heteroresistance to daptomycin has been reported [89]. In a randomised study, resistant post-treatment isolates were found in $7 / 53$ patients (13.2\%) with S. aureus bacteraemia/endocarditis treated with vancomycin compared with $7 / 120$ patients (5.8\%) treated with daptomycin [90]. Selection of vancomycin resistance during vancomycin therapy can confer daptomycin cross-resistance [91-93]. The daptomycin MIC should therefore be checked when this agent is used in patients who have previously received vancomycin therapy [14]. Although the mechanisms of daptomycin resistance are not clear, resistance and clinical failure have been linked to sequential mutations and various changes in membrane structure and function (including drug binding) [69,87,94], analogous to vancomycin resistance [82].

According to a post-marketing retrospective registry, the overall rate of clinical failure in 187 patients with confirmed MRSA infections treated with daptomycin was $11 \%$ [95]. According to multivariate analysis of registry data from 1227 patients with $S$. aureus infections, endocarditis, bacteraemia, severe renal dysfunction and diabetes mellitus were significantly and independently associated with higher rates of daptomycin treatment failure [96]. Up until March 2011, we identified 28 well-documented case reports of daptomycin treatment failure in MRSA infections associated with the development of daptomycin resistance [97-101]. Treatment failure is generally associated with: the presence of significant co-morbidities; serious, high bacterial load infections (e.g. endocarditis); delay in drainage or removal of the infected focus; persistent bacteraemia or recurrence despite glycopeptide therapy; and the use of relatively low daptomycin doses (i.e. $\leq 6 \mathrm{mg} / \mathrm{kg} /$ day). Daptomycin MICs typically rose from $0.5 \mathrm{mg} / \mathrm{L}$ to $4-8 \mathrm{mg} / \mathrm{L}$. The number of cases is small in light of the total usage of daptomycin. However, it is likely that other cases have gone unreported owing to the difficulties involved in full documentation. Measures that may reduce the risk of daptomycin failure include prompt and effective drainage or removal of abscesses, prompt initiation of therapy, and dosing at $10-12 \mathrm{mg} / \mathrm{kg} / \mathrm{day}$. In future it might be possible to use TDM to optimise daptomycin exposure, but no recommendation is possible at present.

Options for the treatment of infections caused by organisms with reduced susceptibility to both vancomycin and daptomycin include quinupristin/dalfopristin, SXT, linezolid or telavancin, possibly in combination with other agents [27].

\section{Conclusions}

Significant developments have taken place in recent years in our understanding of the pathogenicity of $S$. aureus and MRSA infections and the development of resistance in these organisms. In addition, new clinical data are now available to provide better guidance on the use of existing and newly available antibiotics. These data make clearer the increasing limitations of the older glycopeptides and underscore the need for additional new agents.

The major obstacle to the development of new antibiotics for MRSA infections is the need for novel molecular targets. Whilst extant classes of agents effective against MRSA currently provide a variety of options for therapy for the majority of MRSA infections, history shows us that $S$. aureus may develop widespread resistance to any, and perhaps all, of them. As highlighted by the IDSA (in its ' $10 \times 20^{\prime}$ initiative) and other bodies, novel targets must be identified with some regularity or we will face the risk of untreatable $S$. aureus infections.

\section{Acknowledgment}

The authors acknowledge the assistance of Lee Baker (Chester, UK) in the development of this paper.

Funding: National Institutes of Health (to MZD); Funds for Health of Spain (FIS) (to JG); the laboratory of GL has received 
research grants from Europe (FP7-HEALTH-2007, EC 222718), the French Government (ANR 2010-EMMA-027-02), FINOVI Foundation (FINOVI 2010), Pfizer and Novartis.

Competing interests: Current personal interests of IMG include: consultancy - Phico, GSK, MSD, Cubist, Becton Dickinson, Johnson \& Johnson, Astellas, Novartis, Pfizer and bioMérieux; and lecture fees - GSK, MSD, Zeneca, Cubist, Novartis and Pfizer. In his capacity as Secretary General of the International Society of Chemotherapy (ISC), IMG seeks meeting support from a wide range of diagnostic and pharmaceutical companies, including many involved in the manufacture of diagnostics and antibiotics for MRSA; MZD has been awarded a young investigator grant from Pfizer for 2011-2012; JG; has received funds for speaking, consultancy, advisory board membership and travel from Novartis, GSK, AstraZeneca, Astellas, Pfizer and Bayer; SE, JG, TM and GP declare no competing interests.

Ethical approval: Not required.

\section{References}

[1] European Centre for Disease Prevention and Control/European Medicines Agency (ECDC/EMEA). Technical report. The bacterial challenge: time to react. Stockholm, Sweden: ECDC/EMEA; 2009. http://ecdc.europa.eu/en/ publications/Publications/0909_TER_The_Bacterial_Challenge_Time_to_React. pdf [accessed 21.07.11]

[2] European Centre for Disease Prevention and Control (ECDC). Annual epidemiological report on communicable diseases in Europe 2009. Stockholm, Sweden: ECDC; 2010. http://www.ecdc.europa.eu/en/publications/ Publications/0910_SUR_Annual_Epidemiological_Report_on_Communicable Diseases_in_Europe.pdf [accessed 14.06.11].

[3] de Kraker ME, Wolkewitz M, Davey PG, Koller W, Berger J, Nagler J, et al. Clinical impact of antimicrobial resistance in European hospitals: excess mortality and length of hospital stay related to methicillin-resistant Staphylococcus aureus bloodstream infections. Antimicrob Agents Chemother 2011;55:1598-605.

[4] Cosgrove SE, Sakoulas G, Perencevich EN, Schwaber MJ, Karchmer AW, Carmeli Y. Comparison of mortality associated with methicillin-resistant and methicillin-susceptible Staphylococcus aureus bacteremia: a meta-analysis. Clin Infect Dis 2003:36:53-9.

[5] Shurland S, Zhan M, Bradham DD, Roghmann MC. Comparison of mortality risk associated with bacteremia due to methicillin-resistant and methicillin-susceptible Staphylococcus aureus. Infect Control Hosp Epidemiol 2007;28:273-9.

[6] Athanassa Z, Siempos II, Falagas ME. Impact of methicillin resistance on mortality in Staphylococcus aureus VAP: a systematic review. Eur Respir J 2008;28:625-32.

[7] Skov RL, Jensen KS. Community-associated meticillin-resistant Staphylococcus aureus as a cause of hospital-acquired infections. J Hosp Infect 2009:73:364-70.

[8] David MZ, Daum RS. Community-associated methicillin-resistant Staphylococcus aureus: epidemiology and clinical consequences of an emerging epidemic. Clin Microbiol Rev 2010;23:616-87.

[9] Valsesia G, Rossi M, Bertschy S, Pfyffer GE. Emergence of SCCmec type IV and SCCmec type V methicillin-resistant Staphylococcus aureus containing the Panton-Valentine leukocidin genes in a large academic teaching hospital in central Switzerland: external invaders or persisting circulators? J Clin Microbiol 2010;48:720-7.

[10] International Working Group on the Classification of Staphylococcal Cassette Chromosome Elements (IWG-SCC). Classification of staphylococcal cassette chromosome mec (SCCmec): guidelines for reporting novel SCCmec elements. Antimicrob Agents Chemother 2009;53:4961-7.

[11] McDougal LK, Fosheim GE, Nicholson A, Bulens SN, Limbago BM, Shearer JE, et al. Emergence of resistance among USA300 methicillin-resistant Staphylococcus aureus isolates causing invasive disease in the United States. Antimicrob Agents Chemother 2010;54:3804-11.

[12] Li S, Skov RL, Han X, Larsen AR, Larsen J, Sørum M, et al. Novel types of staphylococcal cassette chromosome mec elements identified in clonal complex 398 methicillin-resistant Staphylococcus aureus strains. Antimicrob Agents Chemother 2011;55:3046-50.

[13] Garau J, Bouza E, Chastre J, Gudiol F, Harbarth S. Management of methicillin-resistant Staphylococcus aureus infections. Clin Microbiol Infect 2009;15:125-36.

[14] Gould IM, Cauda R, Esposito S, Gudiol F, Mazzei T, Garau J. Management of serious meticillin-resistant Staphylococcus aureus infections: what are the limits? Int J Antimicrob Agents 2011;37:202-9.

[15] Kuehnert MJ, Kruszon-Moran D, Hill HA, McQuillan G, McAllister SK, Fosheim G, et al. Prevalence of Staphylococcus aureus nasal colonization in the United States, 2001-2002. J Infect Dis 2006;193:172-9.

[16] Lina G, Piémont Y, Godail-Gamot F, Bes M, Peter MO, Gauduchon $\mathrm{V}$, et al. Involvement of Panton-Valentine leukocidin-producing
Staphylococcus aureus in primary skin infections and pneumonia. Clin Infect Dis 1999;29:1128-32.

[17] Gillet Y, Issartel B, Vanhems P, Fournet JC, Lina G, Bes M, et al. Association between Staphylococcus aureus strains carrying gene for Panton-Valentine leukocidin and highly lethal necrotising pneumonia in young immunocompetent patients. Lancet 2002;359:753-9.

[18] Bocchini CE, Hulten KG, Mason Jr EO, Gonzalez BE, Hammerman WA, Kaplan SL. Panton-Valentine leukocidin genes are associated with enhanced inflammatory response and local disease in acute hematogenous Staphylococcus aureus osteomyelitis in children. Pediatrics 2006;117:433-40.

[19] Löffler B, Hussain M, Grundmeier M, Brück M, Holzinger D, Varga G, et at. Staphylococcus aureus Panton-Valentine leukocidin is a very potent cytotoxic factor for human neutrophils. PLoS Pathog 2010;6:e1000715.

[20] Lipinska U, Hermans K, Meulemans L, Dumitrescu O, Badiou C, Duchateau L. et al. Panton-Valentine leukocidin does play a role in the early stage of Staphylococcus aureus skin infections: a rabbit model. PLoS ONE 2011;6:e22864.

[21] Crémieux AC, Dumitrescu O, Lina G, Vallee C, Côté JF, Muffat-Joly M, et al Panton-Valentine leukocidin enhances the severity of community-associated methicillin-resistant Staphylococcus aureus rabbit osteomyelitis. PLoS ONE 2009;4:e7204.

[22] Diep BA, Chan L, Tattevin P, Kajikawa O, Martin TR, Basuino L, et al. Polymorphonuclear leukocytes mediate Staphylococcus aureus Panton-Valentine leukocidin-induced lung inflammation and injury. Proc Natl Acad Sci USA 2010;107:5587-92.

[23] Hirschhausen N, Schlesier T, Schmidt MA, Götz F, Peters G, Heilmann C. A novel staphylococcal internalization mechanism involves the major autolysin Atl and heat shock cognate protein $\mathrm{Hsc70}$ as host cell receptor. Cell Microbiol 2010;12:1746-64.

[24] Grundmeier M, Tuchscherr L, Brück M, Viemann D, Roth J, Willscher E, et al. Staphylococcal strains vary greatly in their ability to induce an inflammatory response in endothelial cells. J Infect Dis 2010;201:871-80.

[25] Tuchscherr L, Löffler B, Buzzola FR, Sordelli DO. Staphylococcus aureus adaptation to the host and persistence: role of loss of capsular polysaccharide expression. Future Microbiol 2010;5:1823-32.

[26] Tuchscherr L, Medina E, Hussain M, Völker W, Heitmann V, Niemann S, et al. Staphylococcus aureus phenotype switching: an effective bacterial strategy to escape host immune response and establish a chronic infection. EMBO Mol Med 2011;3:129-41.

[27] Liu C, Bayer A, Cosgrove SE, Daum RS, Fridkin SK, Gorwitz RJ, et al. Clinical practice guidelines by the Infectious Diseases Society of America for the treatment of methicillin-resistant Staphylococcus aureus infections in adults and children. Clin Infect Dis 2011:52:e18-55.

[28] Gould FK, Brindle R, Chadwick PR, Fraise AP, Hill S, Nathwani D, et al. Guidelines (2008) for the prophylaxis and treatment of methicillin-resistant Staphylococcus aureus (MRSA) infections in the United Kingdom. J Antimicrob Chemother 2009;63:849-61.

[29] Falagas ME, Siempos II, Vardakas KZ. Linezolid versus glycopeptide or $\beta$ lactam for treatment of Gram-positive bacterial infections: meta-analysis of randomised controlled trials. Lancet Infect Dis 2008;8:53-66.

[30] Beibei L, Yun C, Mengli C, Nan B, Xuhong Y, Rui W. Linezolid versus vancomycin for the treatment of Gram-positive bacterial infections: meta-analysis of randomised controlled trials. Int J Antimicrob Agents 2010;35:3-12.

[31] Kalil AC, Murthy MH, Hermsen ED, Neto FK, Sun J, Rupp ME. Linezolid versus vancomycin or teicoplanin for nosocomial pneumonia: a systematic review and meta-analysis. Crit Care Med 2010;38:1802-8.

[32] Walkey AJ, O'Donnell MR, Wiener RS. Linezolid vs glycopeptide antibiotics for the treatment of suspected methicillin-resistant Staphylococcus aureus nosocomial pneumonia: a meta-analysis of randomized controlled trials. Chest 2011;139:1148-55.

[33] Kunkel M, Chastre JE, Kollef M, Niederman M, Shorr AF, Wunderink RG, et al. Linezolid vs vancomycin in the treatment of nosocomial pneumonia proven due to methicillin-resistant Staphylococcus aureus. In: Infectious Diseases Society of America (IDSA) 48th annual meeting. 2010 [Abstract LB-49].

[34] Dodds TJ, Hawke CI. Linezolid versus vancomycin for MRSA skin and soft tissue infections (systematic review and meta-analysis). ANZ J Surg 2009;79:629-35.

[35] Bounthavong M, Hsu DI. Efficacy and safety of linezolid in methicillinresistant Staphylococcus aureus (MRSA) complicated skin and soft tissue infection (cSSTI): a meta-analysis. Curr Med Res Opin 2010;26: $407-21$.

[36] Logman JF, Stephens J, Heeg B, Haider S, Cappelleri J, Nathwani D, et al. Comparative effectiveness of antibiotics for the treatment of MRSA complicated skin and soft tissue infections. Curr Med Res Opin 2010;26:1565-78.

[37] Itani KM, Dryden MS, Bhattacharyya H, Kunkel MJ, Baruch AM, Weigelt JA. Efficacy and safety of linezolid versus vancomycin for the treatment of complicated skin and soft-tissue infections proven to be caused by methicillin-resistant Staphylococcus aureus. Am J Surg 2010;199:804-16.

[38] Novartis. Cubicin. Summary of product characteristics. Novartis; 2006.

[39] Rehm SJ, Boucher H, Levine D, Campion M, Eisenstein BI, Vigliani GA, et al Daptomycin versus vancomycin plus gentamicin for treatment of bacteraemia and endocarditis due to Staphylococcus aureus: subset analysis of patients infected with methicillin-resistant isolates. J Antimicrob Chemother 2008;62:1413-21.

[40] Chaftari AM, Hachem R, Mulanovich V, Chemaly RF, Adachi J, Jacobson K, et al. Efficacy and safety of daptomycin in the treatment of Gram-positive 
catheter-related bloodstream infections in cancer patients. Int J Antimicrob Agents 2010;36:182-6.

[41] Seaton RA, Daptomycin:. rationale and role in the management of skin and soft tissue infections. J Antimicrob Chemother 2008;62(Suppl. 3):iii15-23.

[42] Moise PA, Hershberger E, Amodio-Groton MI, Lamp KC. Safety and clinical outcomes when utilizing high-dose $(\geq 8 \mathrm{mg} / \mathrm{kg}$ ) daptomycin therapy. Ann Pharmacother 2009;43:1211-9.

[43] Utili R, Dohmen P, Dailiana Z, Nacinovich F, Almirante B, Galloway A, et al. Evaluation of safety and tolerability of daptomycin doses $\geq 8 \mathrm{mg} / \mathrm{kg} /$ day: results from 270 patients in the European Cubicin ${ }^{\circledR}$ Outcomes Registry and Experience (EU-CORE ${ }^{\mathrm{sm}}$ ). In: 21st European Congress of Clinical Microbiology and Infectious Diseases (ECCMID)/27th International Congress on Chemotherapy (ICC). 2011 [Abstract P1538].

[44] Bassetti M, Nicco E, Ginocchio F, Ansaldi F, de Florentiis D, Viscoli C. High-dose daptomycin in documented Staphylococcus aureus infections. Int J Antimicrob Agents 2010;36:459-61.

[45] Figueroa DA, Mangini E, Amodio-Groton M, Vardianos B, Melchert A, Fana $C$, et al. Safety of high-dose intravenous daptomycin treatment: three-year cumulative experience in a clinical program. Clin Infect Dis 2009;49:177-80.

[46] Wyeth Pharmaceuticals Inc. Tygacil. Full prescribing information. Wyeth Pharmaceuticals Inc.; 2010.

[47] Wyeth Europa Ltd. Tygacil. Summary of product characteristics. Wyeth Europa Ltd.; 2010.

[48] Freire AT, Melnyk V, Kim MJ, Datsenko O, Dzyublik O, Glumcher F, et al. Comparison of tigecycline with imipenem/cilastatin for the treatment of hospital-acquired pneumonia. Diagn Microbiol Infect Dis 2010;68:140-51.

[49] Cai Y, Wang R, Liang B, Bai N, Liu Y. Systematic review and meta-analysis of the effectiveness and safety of tigecycline for treatment of infectious disease. Antimicrob Agents Chemother 2011;55:1162-72.

[50] European Medicines Agency (EMEA). Questions and answers on the review of Tygacil (tigecycline). Outcome of a renewal procedure. London, UK: EMEA; 2011 [EMA/121925/2011] http://www.ema.europa.eu/docs/ en_GB/document_library/Medicine_QA/human/000644/WC500102228.pdf [accessed 21.07.11].

[51] Pfizer. Direct healthcare professional communication on increase in mortality in clinical trials of Tygacil ${ }^{\circledR}$ (tigecycline). Pfizer; 2011.

[52] Theravance Inc. Vibativ ${ }^{\circledR}$ (telavancin) for injection, for intravenous useinitial U.S. approval: 2009. San Francisco, CA: Theravance Inc.; 2009, http://www.accessdata.fda.gov/drugsatfda_docs/label/2009/022110s000lbl. pdf [accessed 27.07.11].

[53] Stryjewski ME, Graham DR, Wilson SE, O’Riordan W, Young D, Lentnek A et al. Telavancin versus vancomycin for the treatment of complicated skin and skin-structure infections caused by Gram-positive organisms. Clin Infect Dis 2008;46:1683-93.

[54] Rubinstein E, Lalani T, Corey GR, Kanafani ZA, Nannini EC, Rocha MG, et al. Telavancin versus vancomycin for hospital-acquired pneumonia due to Gram-positive pathogens. Clin Infect Dis 2011;52:31-40.

[55] Committee for Medicinal Products for Human Use (CHMP). Summary of opinion (initial authorisation): Vibativ-telavancin. European Medicines Agency; 19 May 2011 [EMA/CHMP/399709/2011] http://www.ema.europa.eu/docs/ en_GB/document_library/Summary_of_opinion_-_Initial_authorisation/ human/001240/WC500106519.pdf [accessed 26.07.11].

[56] Forest Laboratories. Teflaro ${ }^{\circledR}$ prescribing information. Forest Laboratories; 2010.

[57] Corey GR, Wilcox MH, Talbot GH, Thye D, Friedland D, Baculik T. CANVAS 1 investigators. CANVAS 1 , the first phase III, randomized, double-blind study evaluating ceftaroline fosamil for the treatment of patients with complicated skin and skin structure infections. J Antimicrob Chemother 2010;65(Suppl. 4):iv41-51.

[58] Wilcox MH, Corey GR, Talbot GH, Thye D, Friedland D, Baculik T. CANVAS 2 investigators. CANVAS 2, the second phase III, randomized, double-blind study evaluating ceftaroline fosamil for the treatment of patients with complicated skin and skin structure infections. J Antimicrob Chemother 2010;65(Suppl. 4):iv53-65

[59] Corey GR, Wilcox M, Talbot GH, Friedland HD, Baculik T, Witherell GW, et al Integrated analysis of CANVAS 1 and 2: phase 3, multicenter, randomized, double-blind studies to evaluate the safety and efficacy of ceftaroline versus vancomycin plus aztreonam in complicated skin and skin-structure infection. Clin Infect Dis 2010;51:641-50.

[60] European Medicines Agency. Questions and answers on the withdrawal of the marketing authorisation application for Ramvocid (oritavancin). London, UK: EMEA; 24 September 2009 [EMEA/554626/2009].

[61] Dunbar LM, Milata J, Fitzpatrick M, Larrison A, McClure T, Wasilewski MM. Efficacy of oritavancin at single or infrequent doses for the treatment of complicated skin and skin-structure infections (Abstract P1849). Clin Microbiol Infect 2009;15(Suppl. 4):S537

[62] Lauderdale TL, Shiau YR, Lai JF, Chen HC, King CH. Comparative in vitro activities of nemonoxacin (TG-873870), a novel nonfluorinated quinolone, and other quinolones against clinical isolates. Antimicrob Agents Chemother 2010;54:1338-42.

[63] Li CR, Li Y, Li GQ Yang XY, Zhang WX, Lou RH, et al. In vivo antibacterial activity of nemonoxacin, a novel non-fluorinated quinolone. J Antimicrob Chemother 2010;65:2411-5

[64] Hait H, Arbeit R, Molnar D, Noel GJ, Tanaka SK. In a phase 2 complicated skin and soft tissue infections (cSSTI) trial outcomes assessed early in the course of therapy were consistent with outcomes assessed 10-17 days after completing therapy for patients treated with either omadacycline (OMC; PTK796) or linezolid. In: 21st European Congress of Clinical Microbiology and Infectious Diseases (ECCMID)/27th International Congress of Chemotherapy (ICC). 2011.

[65] Schmitz GR, Bruner D, Pitotti R, Olderog C, Livengood T, Williams J, et al. Randomized controlled trial of trimethoprim-sulfamethoxazole for uncomplicated skin abscesses in patients at risk for community-associated methicillin-resistant Staphylococcus aureus infection. Ann Emerg Med 2010;56:283-7.

[66] Jung YJ, Koh Y, Hong SB, Chung JW, Ho Choi S, Kim NJ, et al. Effect of vancomycin plus rifampicin in the treatment of nosocomial methicillin-resistant Staphylococcus aureus pneumonia. Crit Care Med 2010;38:175-80.

[67] Rybak MJ, Lomaestro BM, Rotscahfer JC, Moellering RC, Craig WA, Billeter M, et al. Vancomycin therapeutic guidelines: a summary of consensus recommendations from the Infectious Diseases Society of America, the American Society of Health-System Pharmacists, and the Society of Infectious Diseases Pharmacists. Clin Infect Dis 2009;49:325-7.

[68] Mendes RE, Deshpande LM, Castanheira M, DiPersio J, Saubolle MA, Jones RN. First report of $c f r$-mediated resistance to linezolid in human staphylococcal clinical isolates recovered in the United States. Antimicrob Agents Chemother 2008;52:2244-6.

[69] Jones T, Yeaman MR, Sakoulas G, Yang SJ, Proctor RA, Sahl HG, et al. Failures in clinical treatment of Staphylococcus aureus infection with daptomycin are associated with alterations in surface charge, membrane phospholipid asymmetry, and drug binding. Antimicrob Agents Chemother 2008;52:269-78.

[70] Hawser SP, Bouchillon SK, Hoban DJ, Dowzicky M, Babinchak T. Rising incidence of Staphylococcus aureus with reduced susceptibility to vancomycin and susceptibility to antibiotics: a global analysis 2004-2009. Int J Antimicrob Agents 2011;37:219-24.

[71] Sievert DM, Rudrik JT, Patel JB, McDonald LC, Wilkins MJ, Hageman JC. Vancomycin-resistant Staphylococcus aureus in the United States, 2002-2006. Clin Infect Dis 2008;46:668-74

[72] Finks J, Wells E, Dyke TL, Husain N, Plizga L, Heddurshetti R, et al. Vancomycinresistant Staphylococcus aureus, Michigan, USA 2007. Emerg Infect Dis 2009;15:943-5

[73] Saha B, Singh AK, Ghosh A, Bal M. Identification and characterization of a vancomycin-resistant Staphylococcus aureus isolated from Kolkata (South Asia). J Med Microbiol 2008;57:72-9.

[74] Aligholi M, Emaneini M, Jabalameli F, Shahsavan S, Dabiri H, Sedaght H. Emergence of high-level vancomycin-resistant Staphylococcus aureus in the Imam Khomeini Hospital in Tehran. Med Princ Pract 2008;17:432-4.

[75] Moise PA, Smyth DS, El-Fawal N, Robinson DA, Holden PN, Forrest A, et al. Microbiological effects of prior vancomycin use in patients with methicillin-resistant Staphylococcus aureus bacteraemia. J Antimicrob Chemother 2008;61:85-90.

[76] Soriano A, Marco F, Martínez JA, Pisos E, Almela M, Dimova VP, et al. Influence of vancomycin minimum inhibitory concentration on the treatment of methicillin-resistant Staphylococcus aureus bacteremia. Clin Infect Dis 2008;46:193-200.

[77] Musta AC, Riederer K, Shemes S, Chase P, Jose J, Johnson LB, et al. Vancomycin MIC plus heteroresistance and outcome of methicillin-resistant Staphylococcus aureus bacteremia: trends over 11 years. J Clin Microbiol 2009;47: 1640-4.

[78] Khatib R, Jose J, Musta A, Sharma M, Fakih MG, Johnson LB, et al. Relevance of vancomycin-intermediate susceptibility and heteroresistance in methicillin-resistant Staphylococcus aureus bacteraemia. J Antimicrob Chemother 2011;66:1594-9.

[79] van Hal SJ, Paterson DL. Systematic review and meta-analysis of the significance of heterogeneous vancomycin-intermediate Staphylococcus aureus isolates. Antimicrob Agents Chemother 2011;55:405-10.

[80] Thomson AH, Staatz CE, Tobin CM, Gall M, Lovering AM. Development and evaluation of vancomycin dosage guidelines designed to achieve new target concentrations. J Antimicrob Chemother 2009;63:1050-7.

[81] Berthoin K, Ampe E, Tulkens PM, Carryn S. Correlation between free and total vancomycin serum concentrations in patients treated for Gram-positive infections. Int J Antimicrob Agents 2009;34:555-60.

[82] Gould IM. Clinical activity of anti-Gram-positive agents against methicillinresistant Staphylococcus aureus. J Antimicrob Chemother 2011;66(Suppl. 4):iv17-21.

[83] Meka VG, Gold HS. Antimicrobial resistance to linezolid. Clin Infect Dis 2004;39:1010-5.

[84] Sánchez García M, De la Torre MA, Morales G, Peláez B, Tolón MJ, Domingo $\mathrm{S}$, et al. Clinical outbreak of linezolid-resistant Staphylococcus aureus in an intensive care unit. JAMA 2010;303:2260-4.

[85] Morales G, Picazo JJ, Baos E, Candel FJ, Arribi A, Peláez B, et al. Resistance to linezolid is mediated by the $c f r$ gene in the first report of an outbreak of linezolid-resistant Staphylococcus aureus. Clin Infect Dis 2010;50:821-5.

[86] Camargo IL, Neoh HM, Cui L, Hiramatsu K. Serial daptomycin selection generates daptomycin-nonsusceptible Staphylococcus aureus strains with a heterogeneous vancomycin-intermediate phenotype. Antimicrob Agents Chemother 2008;52:4289-99.

[87] Mishra NN, Yang SJ, Sawa A, Rubio A, Nast CC, Yeaman MR et al. Analysis of cell membrane characteristics of in vitro-selected daptomycin-resistant strains of methicillin-resistant Staphylococcus aureus. Antimicrob Agents Chemother 2009:53:2312-8.

[88] Sader HS, Becker HK, Moet GJ, Jones RN. Antimicrobial activity of daptomycin tested against Staphylococcus aureus with vancomycin MIC of $2 \mathrm{~g} / \mathrm{mL}$ isolated 
in the United States and European hospitals (2006-2008). Diagn Microbiol Infect Dis 2010;66:329-31.

[89] Kelley PG, Gao W, Ward PB, Howden BP. Daptomycin non-susceptibility in vancomycin-intermediate Staphylococcus aureus (VISA) and heterogeneousVISA (hVISA): implications for therapy after vancomycin treatment failure. J Antimicrob Chemother 2011;66:1057-60.

[90] Fowler VG, Boucher HW, Corey GR, Abrutyn E, Karchmer AW, Rupp $\mathrm{ME}$, et al. Daptomycin versus standard therapy for bacteremia and endocarditis caused by Staphylococcus aureus. N Engl J Med 2006;355: 653-65.

[91] Cui L, Tominaga E, Neoh HM, Hiramatsu K. Correlation between reduced daptomycin susceptibility and vancomycin resistance in vancomycinintermediate Staphylococcus aureus. Antimicrob Agents Chemother 2006; $50: 1079-82$.

[92] Patel JB, Jevitt LA, Hageman J, McDonald LC, Tenover FC. An association between reduced susceptibility to daptomycin and reduced susceptibility to vancomycin in Staphylococcus aureus. Clin Infect Dis 2006;42: 1652-3.

[93] Sakoulas G, Alder J, Thauvin-Eliopoulos C, Moellering Jr RC, Eliopoulos GM. Induction of daptomycin heterogeneous susceptibility in Staphylococcus aureus by exposure to vancomycin. Antimicrob Agents Chemother 2006;50:1581-5.

[94] Yang SJ, Nast CC, Mishra NN, Yeaman MR, Fey PD, Bayer AS. Cell wall thickening is not a universal accompaniment of the daptomycin nonsusceptibility phenotype in Staphylococcus aureus: evidence for multiple resistance mechanisms. Antimicrob Agents Chemother 2010;54:3079-85.
[95] Gonzalez-Ruiz A, Beiras-Fernandez A, Lehmkuhl H, Seaton RA, Loeffler J, Chaves RL. Clinical experience with daptomycin in Europe: the first 2.5 years. J Antimicrob Chemother 2011;66:912-9.

[96] Sakoulas G, Brown J, Lamp KC, Friedrich LV, Lindfield KC. Clinical outcomes of patients receiving daptomycin for the treatment of Staphylococcus aureus infections and assessment of clinical factors for daptomycin failure: a retrospective cohort study utilizing the Cubicin Outcomes Registry and Experience. Clin Ther 2009;31:1936-45.

[97] Mangili A, Bica I, Snydman DR, Hamer DH. Daptomycin-resistant, methicillinresistant Staphylococcus aureus bacteremia. Clin Infect Dis 2005;40:1058-60.

[98] Enoch DA, Phillimore N, Karas JA, Horswill L, Mlangeni DA. Relapse of enterococcal prosthetic valve endocarditis with aortic root abscess following treatment with daptomycin in a patient not fit for surgery. J Med Microbiol 2010;59:482-5.

[99] Jacobson LM, Milstone AM, Zenilman J, Carroll KC, Arav-Boger R. Daptomycin therapy failure in an adolescent with methicillin-resistant Staphylococcus aureus bacteremia. Pediatr Infect Dis J 2009;28:445-7.

[100] van Hal SJ, Paterson DL, Gosbell IB. Emergence of daptomycin resistance following vancomycin-unresponsive Staphylococcus aureus bacteraemia in a daptomycin-naïve patient - a review of the literature. Eur J Clin Microbiol Infect Dis 2011;30:603-10.

[101] Boyle-Vavra S, Jones M, Gourley BL, Holmes M, Ruf R, Balsam AR, et al. Comparative genome sequencing of an isogenic pair of USA800 clinical methicillin-resistant Staphylococcus aureus isolates obtained before and after daptomycin treatment failure. Antimicrob Agents Chemother 2011;55:2018-25. 